\title{
Crystallographically uniform arrays of ordered (In)GaN nanocolumns
}

\author{
Ž. Gačević, A. Bengoechea-Encabo, S. Albert, A. Torres-Pardo, \\ J. M. González-Calbet, and E. Calleja
}

\begin{abstract}
In this work, through a comparative study of self-assembled (SA) and selective area grown (SAG) (In)GaN nanocolumn (NC) ensembles, we first give a detailed insight into improved crystallographic uniformity (homogeneity of crystallographic tilts and twists) of the latter ones. The study, performed making use of: reflective high energy electron diffraction, X-ray diffraction and scanning electron microscopy, reveals that unlike their SA counterparts, the ensembles of SAG NCs show single epitaxial relationship to both sapphire(0001) and $\mathrm{Si}(111)$ underlying substrates. In the second part of the article, making use of X-ray diffraction, we directly show that the selective area growth leads to improved compositional uniformity of InGaN NC ensembles. This further leads to improved spectral purity of their luminescence, as confirmed by comparative macro-photoluminescence measurements performed on SA and SAG InGaN NC ensembles. An improved crystallographic uniformity of NC ensembles facilitates their integration into optoelectronic devices, whereas their improved compositional uniformity allows for their employment in single-color optoelectronic applications.
\end{abstract}

\section{INTRODUCTION}

The high potential of GaN nanocolumns (NCs) was recognized in the late 1990s, when several groups reported their self-assembled (SA) growth and their supreme crystal quality. ${ }^{1,2}$ The SA mode is, however, characterized by intrinsically high randomness, which limits the control of NCs growth (density, size, and orientation) and their consequent processing into devices. To overcome these highly undesired drawbacks, several groups reported realization of ordered arrays of GaN NCs, obtained by means of selective area growth (SAG) homoepitaxy. ${ }^{3-5}$ Apart from improved control over NCs' (i) position and (ii) size (diameter and height), the SAG homoepitaxy also provides dramatically improved control over $\mathrm{NCs}^{\prime}$ (iii) orientation (tilt and twist) and, consequently, (iv) compositional uniformity, when it comes to InGaN NC ensembles. The SAG growth technique thus opens the door for the realization of a wide variety of uniform and scalable NC-based structures and devices, such as (a) arrays of nanolight emitting diodes (nano-LEDs), ${ }^{6-8}$ (b) arrays of quantum light (single photon) emitters, ${ }^{9}$ (c) arrays of gate-all-around nano-transistors, ${ }^{10}$ (d) pseudo-substrates, ${ }^{11}$ and (e) photonic crystals. ${ }^{12}$

When NCs processing into a functional device requires their electrical functionalization (devices (a) and (c)), then it is desirable the $\mathrm{NC}$ epilayer to be merged in the final stage of its growth. In this way, the highly demanding technological task of contacting billions of independent NCs is substituted with much easier one that is, contacting single compact layer. The NC merging step is also the crucial one for the realization of semi- and non-polar pseudo-substrates (d), with improved crystal quality. Merging of mutually tilted and/or twisted (crystallographically non-uniform) nano-crystals leads to the formation of screw, edge and/or mixed dislocations, which deteriorate the layer's crystal quality. ${ }^{13}$ This directly implies that, as long as the NC merging is concerned, the high uniformity of NCs tilts and twists (crystallographic uniformity (iii)), plays a crucial role for their practical applications. The requirement of precise control of NC orientation is not necessarily related to the merging step. In the case of a dot-in-wire quantum light emitter (b), the polarization of emitted photons is influenced by crystallographic orientation of the emitting quantum dot, which is directly inherited from the orientation of the hosting NC. ${ }^{9}$ In a NC-based photonic crystal (e), misaligned NCs introduce local photonic band gap alterations, acting thus as defects in the photonic crystal. ${ }^{12}$

Apart from crystallographic uniformity, another issue starts to play a crucial role when applications come to the employment of InGaN NCs, and that is ensemble compositional uniformity (iv). Namely, when employed as building blocks of light emitting devices ( $a$ and $b$ ), poor compositional uniformity of InGaN NC ensemble leads to its broad emission, which may be incompatible with single-color applications. ${ }^{14-16}$ The highly varying: density, morphology, and orientation of NC ensembles fabricated by SA growth, produce differences in effective $\mathrm{In}, \mathrm{Ga}$, and $\mathrm{N}$ fluxes on $\mathrm{NCs}^{\prime}$ tops, resulting with formation of compositionally different columnar InGaN sections (from one NC to another). The high uniformity in $\mathrm{NCs}^{\prime}$ density, size, and orientation, which is guaranteed by the SAG homoepitaxy, on the other hand, leads to their compositional uniformity and, consequently, to improved spectral purity of the ensemble luminescence. ${ }^{68}$

The arrays of SAG NCs are already being processed into structures and devices that are not available within the limitations of the SA growth. In our recent publications, we 
reported realization of: arrays of blue, green, and yellow nano-LEDs, ${ }^{8}$ arrays of blue and green quantum light emitters, ${ }^{9}$ as well as GaN semi-polar pseudo-substrates. ${ }^{11}$ In this work, we provide a detailed insight into those properties of SAG homoepitaxy that allowed for these achievements. As previously mentioned, due to their supreme importance, the focus of the work is on NC ensemble crystallographic and compositional uniformity.

\section{EXPERIMENTAL}

The samples under study were grown in a RIBER Compact 21 Molecular Beam Epitaxy (MBE) system, equipped with a radio-frequency plasma nitrogen source and standard Knudsen cells for Ga and In. The SAG NC samples were grown on $\mathrm{GaN}$-on-Si(111) and GaN-on-sapphire(0001) templates. In the case of the GaN-on-Si(111) templates, $200-500 \mathrm{~nm}$ thick GaN were grown by MBE on two-inch Si(111) substrates with a $\sim 4^{\circ}$ miscut towards (11-2) crystallographic direction. More details on the GaN buffer growth can be found elsewhere. ${ }^{17}$ Commercial $\sim 3.3 \mu \mathrm{m}$ thick GaNon-sapphire templates (Lumilog) were grown by metal organic chemical vapor deposition (MOCVD). On both types of templates, the SAG of GaN NCs was achieved by using $\sim 7 \mathrm{~nm}$ thick Ti mask fabricated by colloidal lithography. The Ti mask consists of uniform nanoholes arranged in a compact hexagonal lattice with a $\sim 270 \mathrm{~nm}$ pitch. The nanoholes' diameters can be varied in a controlled way from $\sim 220$ down to $\sim 100 \mathrm{~nm}$. Details about colloidal lithography can be found in our previous publications. ${ }^{18}$ After the colloidal mask was prepared, the SAG GaN NCs were grown at $\sim 840^{\circ} \mathrm{C}$, with impinging fluxes $\Phi_{\mathrm{Ga}} \sim 18 \mathrm{~nm} / \mathrm{min}$ and $\Phi_{\mathrm{N}} \sim 5 \mathrm{~nm} / \mathrm{min}^{5}$

The SA GaN NCs were grown on bare Si (111) substrates. Prior to the growth, the Si wafers were outgassed in the growth chamber for $30 \mathrm{~min}$ and at $860^{\circ} \mathrm{C}$, to remove surface oxide. The SA GaN NCs growth was then performed at $\sim 800^{\circ} \mathrm{C}$, under highly $N$-rich conditions: $\Phi_{\mathrm{Ga}} \sim 4 \mathrm{~nm} / \mathrm{min}$ and $\Phi_{\mathrm{N}} \sim 14 \mathrm{~nm} / \mathrm{min}^{19}$

Finally, the growth of columnar InGaN sections on the top of both SAG and SA GaN NCs, was performed at lower growth temperatures, under N-rich conditions. To achieve InGaN over full compositional range, the growth temperature was varied between 300 and $650{ }^{\circ} \mathrm{C}$, whereas the impinging flux ratio $\Phi_{\mathrm{In}} /\left(\Phi_{\mathrm{Ga}}+\Phi_{\mathrm{In}}\right)$ was varied from 0.4 to 1 . Further growth details about this growth step can be found in our previous publications. ${ }^{19,20}$

The morphological characterization of NCs has been performed with a Crestec CABL-9500C scanning electron microscope (SEM). To study crystallographic uniformity of GaN $\mathrm{NC}$ ensembles, surface diffraction patterns, obtained by reflective high energy electron diffraction (RHEED), were employed. The diffraction patterns were obtained at $15 \mathrm{keV}$ on a fluorescent screen and were recorded by a charge-couple device camera. The information about NCs crystal quality (and other relevant structural features, such as polarity and strain) was acquired with an aberration-corrected scanning transmission electron microscopy (STEM) JEOLJEMARM200cF, with atomic spatial resolution. To study crystallographic and compositional uniformity of InGaN NC ensembles (as well as their other relevant properties, such as epitaxial relationship and strain) volume diffraction measurements, realized with X-ray diffraction (XRD) technique, were employed. The measurements were performed with $\mathrm{Cu}$ $K \alpha I$ line $\left(\lambda_{X}=1.5406 A\right)$, in a commercial Panalytical X'Pert Pro diffractometer equipped with a $\mathrm{Ge}(220)$ hybrid monochromator. Finally, optical quality of the samples was assessed by photoluminescence (PL) measurements, exciting with a $\mathrm{HeCd}$ laser $(\lambda=325 \mathrm{~nm})$ with a power density of $\sim 1$ $\mathrm{W} \mathrm{cm}{ }^{-2}$ at $\sim 10 \mathrm{~K}$.

\section{RESULTS}

In Sec. III A, we analyze ensembles of $\mathrm{GaN}$ NCs. Growth details about this sample series can be found in our previous publications. $^{5,19}$ Through a comparative study between ensembles of SA and SAG GaN NCs, we first explain the mechanisms of the SAG homoepitaxy which lead to: ordered NC ensembles (site-control), with a good morphological uniformity (size-control) and a good crystallographic uniformity (orientation-control). This study is performed by the combination of SEM and RHEED. Second, we provide detailed insights into two other NCs properties, which are crucial for their integration into optoelectronic devices: NCs' crystal quality, assessed by STEM, and $\mathrm{NCs}^{\prime}$ optical quality, assessed by macro-PL.

In Sec. III B, we analyze ensembles of $\operatorname{In}(\mathrm{Ga}) \mathrm{N}$ NCs. Growth details about this sample series can be found in our previous publications. ${ }^{19-21}$ Through a comparative study between ensembles of SA and SAG InGaN NCs, we first address the benefits of the SAG, by combination of SEM and XRD. Second, we provide a detailed insight into columnar InGaN compositional uniformity, which is assessed by XRD technique and complemented with macro-PL measurements.

\section{A. Ordered ensembles of GaN nanowires}

Crystalline properties of a reference SA GaN NCs grown heteroepitaxially on bare $\mathrm{Si}(111)$ (sample $\mathrm{A} 1)^{19}$ are compared with two samples of ordered GaN NCs grown by SAG homoepitaxy on GaN-on-Si(111) and GaN-on-sapphire(0001) templates (samples A2 and A3, respectively). ${ }^{5}$ Details about these samples (series A) are summarized in Table I.

\section{SA heteroepitaxy vs. SAG homoepitaxy: Site-, size-, and orientation-control}

Figure 1 shows top and lateral SEM view of the studied samples.

The SA heteroepitaxy (Figure 1(A1)) is characterized with a random self-nucleation mechanism and a weak epitaxial constraint. The self-nucleation mechanism (the details of which can be found in a recent publication of Consonni et $a .^{22}$ ) leads to: (i) a random $\mathrm{NC}$ positioning (no site control) and (ii) a low uniformity of NC diameters (poor size control). In the early growth stage, the underlying $\mathrm{Si}(111)$ substrate is (intentionally or spontaneously) nitridated, resulting with a formation of a $2-3 \mathrm{~nm}$ thick amorphous $\mathrm{SiN}$ layer on the substrate surface. A nucleation of initial $\mathrm{GaN}$ 
TABLE I. Summary of the studied GaN NC samples (series A). ${ }^{5,19}$ The buffer thickness and NCs heights are estimated by SEM.

\begin{tabular}{lccc}
\hline \hline GaN samples & Substrate & $\begin{array}{c}\text { GaN buffer } \\
\text { thickness }(\mu \mathrm{m})\end{array}$ & $\begin{array}{c}\text { GaN NCs } \\
\text { height }(\mu \mathrm{m})\end{array}$ \\
\hline A1 $^{5}$ & $\mathrm{Si}(111)$ & $\ldots$ & 1 \\
Ref. SA NCs & GaN-on-Si(111) & 0.5 & 0.25 \\
$\begin{array}{l}\text { A2 } \\
\text { SAG NCs }\end{array}$ & GaN-on-sapphire(0001) & 3.3 & 0.6 \\
A3 $^{19}$ & & & \\
SAG NCs & & & \\
\hline
\end{tabular}

nano-islands (seeds) thus proceeds on this amorphous layer, i.e., without direct crystal-to-crystal contact (weak epitaxial constraint) with the underlying Si. This results with a high dispersion of GaN seeds' tilts and twists, and, consequently with a (iii) low crystallographic uniformity of NCs developed from them in the later growth stage (poor orientation control).

The SAG homoepitaxy (Figures 1(A2) and 1(A3)) is on the other hand characterized with a selective GaN growth and a direct homoepitaxial crystal-to-crystal contact. The selective GaN growth (the details of which can be found in our separate publication ${ }^{23}$ ) leads to: (i) a precise NC positioning (site control), and (ii) a high uniformity of NC diameters (good size control). Since the nucleation of initial GaN seeds proceeds directly on the underlying GaN crystal, their tilts and twists are directly determined by the underlying single-crystal GaN buffer. This results with low tilt/twist dispersion and consequently with (iii) a high crystallographic

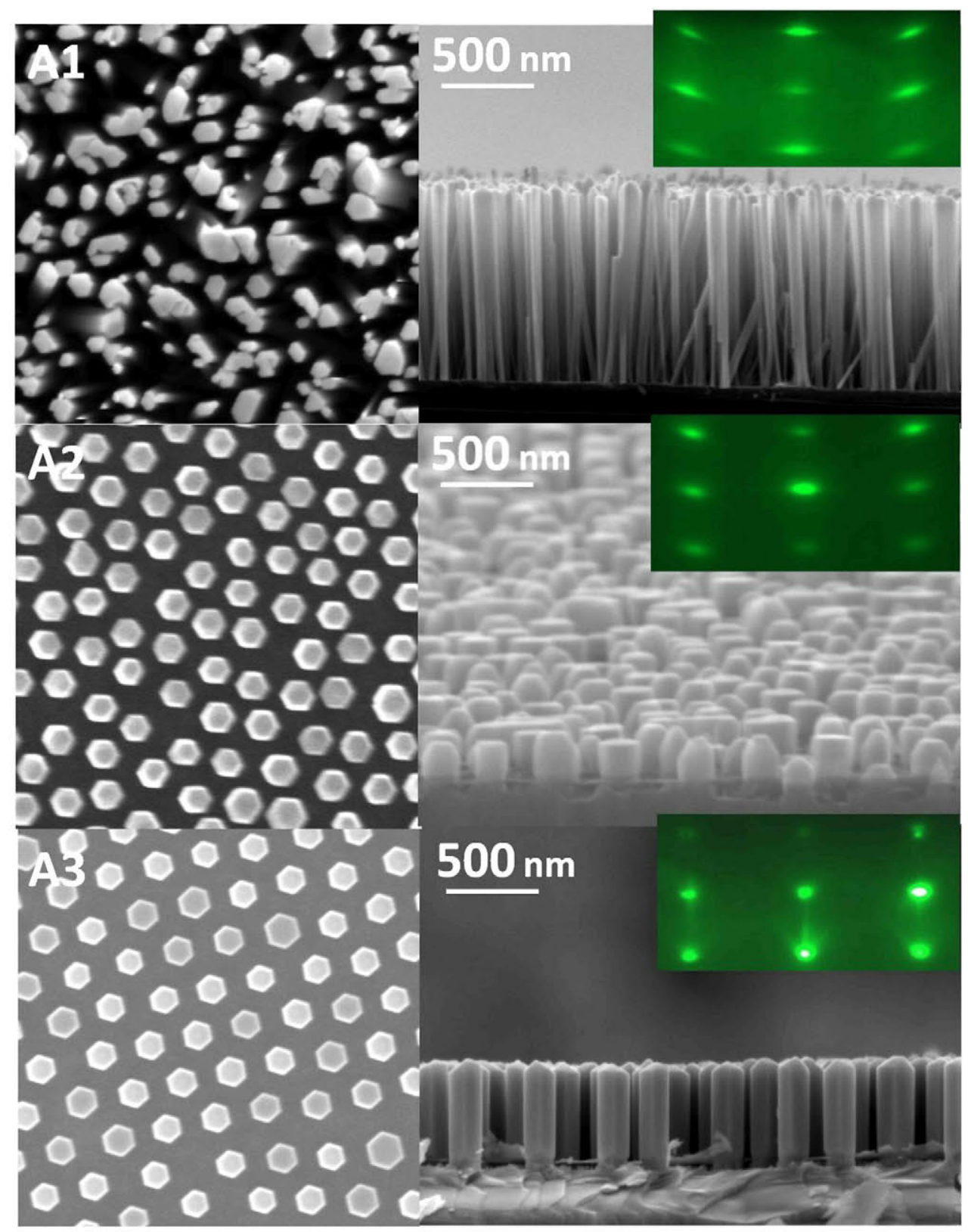

FIG. 1. Top and lateral SEM view of samples: A1, A2, and A3. The insets represent the corresponding RHEED patterns, taken in-situ along (11-20) azimuth. (A1) The SA heteroepitaxy is characterized with: (i) no site control, (ii) a poor size control, and (iii) a poor orientation control. ((A2) and (A3)) The SAG homoepitaxy is, on the other hand, characterized with: (i) a site control, (ii) a good size control, and (iii) a good orientation control. 
uniformity of NCs developed from them in the later growth stage (good orientation control).

The insets in Figure 1 represent the corresponding RHEED patterns, taken in-situ along (11-20) azimuth. Since RHEED is a grazing incidence technique, the recorded diffraction patterns originate from those electrons that diffract on $\mathrm{NCs}^{\prime}$ top crystal planes (no diffraction from the underlying substrate). The annular RHEED pattern (A1) is typical for SA heteroepitaxy and is a signature of poor crystallographic uniformity of the analyzed ensemble. When NCs are fabricated by SAG homoepitaxy on $\mathrm{GaN}$ templates, they exhibit bright spotty RHEED patterns ((A2) and (A3)), more evident in sample A3. The spotty RHEED is a signature of 3D electron diffraction through a monocrystal and consequently an indication of a good crystallographic uniformity of the analyzed ensemble. ${ }^{24}$

Figures 1(A1) to 1(A3) clearly show that the SAG homoepitaxy leads to a dramatically improved uniformity in $\mathrm{NCs}^{\prime}$ size. To quantify this improvement, we performed a statistical analysis of the $\mathrm{NCs}^{\prime}$ diameter on samples A1-A3, over $2.2 \times 2.2 \mu \mathrm{m}^{2}$ areas shown in Figure 1 (top SEM view), using free software from Nanotec. ${ }^{25}$ Figures 2(A1)-2(A3) reveal almost one order of magnitude difference of the diameters' standard deviations, when the fabrication method changes from SA heteroepitaxy to SAG homoepitaxy, i.e., $\sigma_{\mathrm{D}}(\mathrm{A} 1) \sim 45 \%, \sigma_{\mathrm{D}}(\mathrm{A} 2) \sim 6 \%$, and $\sigma_{\mathrm{D}}(\mathrm{A} 3) \sim 6 \%$, respectively. Note that the statistical broadening in sample A1 is attributed, on one hand to the random nature of the SA selfnucleation mechanism and, on the other hand, to the NCs tendency to merge (due to ensemble poor crystallographic uniformity) affecting directly the measured diameter. ${ }^{26}$ Neither of the two broadening mechanisms is present in the case of SAG homoepitaxy ((A2) and (A3)). A slight statistical broadenings in the latter cases, which are found identical, are mainly attributed to the size dispersion of the polystyrene nanobeads (estimated at $\sim 5 \%$ ) used for the fabrication of colloidal Ti nanohole mask. ${ }^{27}$

It has to be remarked that, regarding $\mathrm{NCs}^{\prime}$ order and $\mathrm{NCs}^{\prime}$ crystallographic uniformity, slight differences between SAG ensembles grown on Si (A2) and sapphire (A3), are observed (see top SEM images and RHEED, insets in Figure 1). These differences do not arise from the $S A G$ process itself or from the homoepitaxial relationship, which are same in both cases, but rather from the underlying $\mathrm{GaN}$ templates morphology and quality. Indeed, the surface root mean square roughnesses of the two templates differ in nearly one order of magnitude $\sim 10 \mathrm{~nm}$ to $\sim 1 \mathrm{~nm}$, respectively, (estimated by atomic force microscopy over a $10 \times 10 \mu \mathrm{m}^{2}$ area). ${ }^{20} \mathrm{~A}$ smooth surface favors better ordering of the polystyrene nanobeads during colloidal lithography, yielding thus more regular lithographic masks, and consequently better ordered arrays of NCs (Figure 1(A2) vs. 1(A3)). Similarly, the two templates differ in more than one order of magnitude in crystallographic uniformity. ${ }^{28}$ The two templates tilts/twists are found to be $\sim 1^{\circ} / 2^{\circ}$ in the former ( $\mathrm{Si}$ ) and $\sim 0.05^{\circ} / 0.1^{\circ}$ in the latter (sapphire) case (the values estimated by XRD $\omega$-rocking scans around (0002) and $\varphi$-scans around (10-12) Bragg spots, respectively). The superior crystallographic uniformity of the underlying buffer provides better crystallographic uniformity of initial $\mathrm{GaN}$ seeds and consequently better crystallographic uniformity of the $\mathrm{NC}$ ensemble developed from them in the posterior growth stage.

\section{Structural and optical properties of SAG NCS}

For their employment as building blocks of optoelectronic devices, the $\mathrm{GaN} \mathrm{NCs}^{\prime}$ crystal and optical quality play crucial roles. In this section, we address these two issues, making use of STEM and macro-PL characterizations.

a. Structural properties. High angle annular dark field (HAADF) images, acquired on a randomly chosen NC from sample A3, at the upper NC part (Fig. 3(a)) reveal a uniform crystal structure (Fig. 3(b)). The NCs are found free of extended crystal defects. The selective area electron diffraction (SAED, Fig. 3(c)) confirms that the NCs crystallize in wurtzite form. To gain further knowledge about the GaN polarity and its lattice constants, annular bright field images $(\mathrm{ABF})$ with atomic resolution were acquired (Fig. 3(d)). The images confirm that the NCs grow with Ga polarity and with the strain-free " $a$ " and " $c$ " lattice parameters: $\sim 3.19$ and $\sim 5.18 \AA$, respectively, (see Fig. 4(e), for clarity). In summary, the formation of: Ga-polar, strain-free wurtzite $\mathrm{GaN}$ NCs, which are free of extended crystal defects is confirmed.

b. Optical properties. To get insight into NCs optical quality (samples A1-A3), low temperature ( $\sim 10 \mathrm{~K})$ macro-
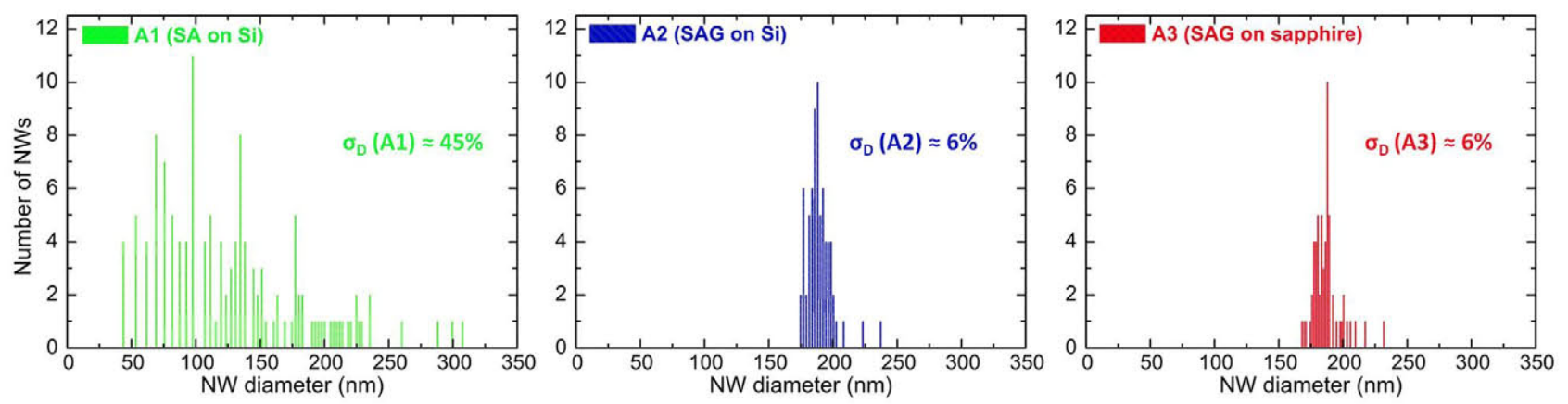

FIG. 2. Statistical analysis of diameters of $\mathrm{NCs}^{\prime}$ diameter (D) has been performed over $2.2 \times 2.2 \mu \mathrm{m}^{2}$ area of samples (A1)-(A3), shown as top SEM view, in Figure 1. The number of analyzed NCs is: 132,70 , and 60 , respectively. The diameter standard deviations $\sigma_{\mathrm{D}}$ are found to be $\sim 45 \%, \sim 6 \%$, and $6 \%$, respectively. 
PL measurements were performed. To avoid interfering PL signals from the GaN buffers, the studied NCs were transferred from their native substrates to optically inactive bare Si substrates (Figs. 4(A1) and 4(A3)). In this experimental setup, around $10^{6} \mathrm{NCs}$ were probed together.

Before we move to PL data analysis, it is worth to notice that PL of a nearly perfect crystal is expected to exhibit following properties:

(i) intense near-band-edge emission,

(ii) narrow near-band-edge emission,

(iii) zero below-band-gap emission.

Due to the high lattice mismatch, the growth of bulk $\mathrm{GaN}$ on commercial substrates is followed by a formation of dense network of threading dislocations at the heterointerface that propagates into the grown bulk material. Consequently, the PL spectra of GaN templates typically show a significant below-band-gap emission. In the present case, $\mathrm{PL}$ of GaN-on-Si (MBE grown, Fig. 4(a)) shows a long below-band-gap tail. PL of GaN-on-sapphire (MOCVD grown), on the other hand, shows a broad violet band (centered around $3.0 \mathrm{eV}$ ) together with the common yellow band (centered around $2.2 \mathrm{eV}$, Fig. 4(a)).

The near-band-edge PL of NCs (samples A1-A3) is typically found between two and four orders of magnitude more intense than that of their bulk counterparts (property (i)). Among the NC samples, however, the PL intensity does not change significantly, being the recorded differences typically within the range of half order of magnitude. The NC samples' near-band-edge PLs are dominated by narrow donorbound exciton line, at $3.471 \mathrm{eV}$ (Fig. 4(b)). The position of this line indicates a zero average strain in all NC samples. ${ }^{29}$ Its width is found as low as $\sim 1.7 \mathrm{meV}$ for sample $\mathrm{A} 3$ (property (ii)), the value that is similar to the narrowest lines reported for SA GaN NCs. ${ }^{2,30,31}$ Finally, below-band-gap emission bands are not present in the $\mathrm{NC}$ samples (condition (iii)).

The previous results suggest that the NCs, grown in either SA or SAG mode, are nano-crystals with exceptional

(a)

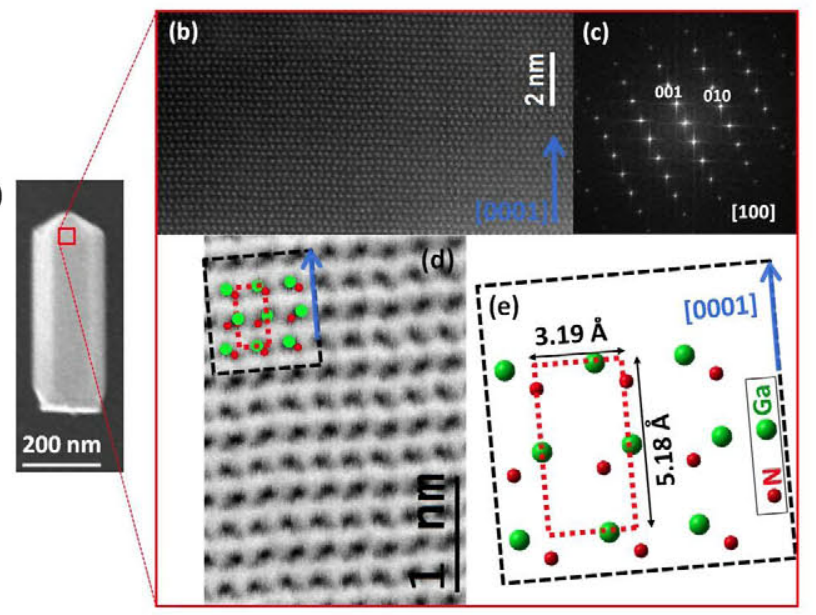

FIG. 3. (a) SEM image of a randomly selected NC from sample A3. STEM: (b) HAADF, (c) SEAD and (d) and (e) ABF images, taken $\sim 30 \mathrm{~nm}$ below the NC tip, confirm uniform Ga polar and strain-free wurtzite structure, free of recurrent crystal defects.
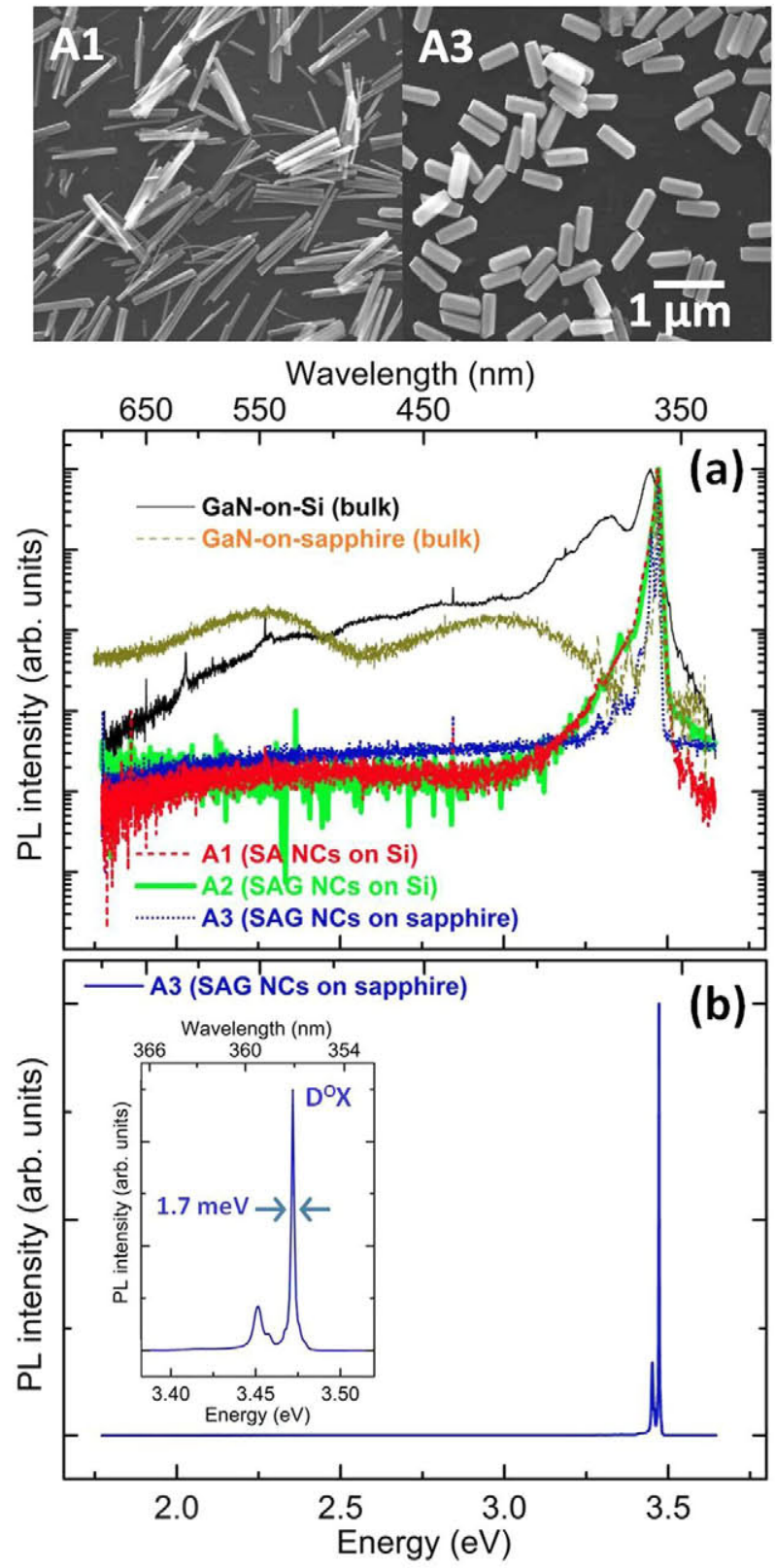

FIG. 4. SEM images of NCs transferred to optically inactive Si substrate (samples A1 and A3). (a) Normalized PL spectra of NC samples A1, A2, and $\mathrm{A} 3$ compared with those of the GaN-on-Si and GaN-on-sapphire pseudosubstrates. (b) PL spectrum of $\sim 10^{6}$ randomly chosen NCs from sample A3, reveal: intense and narrow near-band-edge emission and zero belowband-gap emission. Inset: near-band-edge emission in detail.

quality, in agreement with STEM findings on sample A3. In Fig. 4(b), we show PL spectrum of sample A3, over a wide energy range, and with a linear PL intensity scale. This spectrum gives an approximate idea about optical properties of SAG GaN NCs, when they are integrated as building blocks of optoelectronic devices.

In the end, it is worth to notice that the drastically higher PL intensity of NC samples, in respect to their bulk counterparts, cannot be exclusively attributed to the improved crystal quality, since other parameters, such as different extraction efficiencies of flat bulk layers and dispersed NCs, also play a significant role. 
TABLE II. Summary of the In(Ga)N NC samples (series B). ${ }^{19,20}$ The buffer layers' thicknesses and columnar InGaN sections' heights are estimated by SEM. The columnar InGaN section composition is estimated by XRD.

\begin{tabular}{|c|c|c|c|}
\hline InGaN samples & Substrate & $\begin{array}{c}\text { GaN buffer } \\
\text { thickness }(\mu \mathrm{m})\end{array}$ & $\begin{array}{l}\text { InGaN section } \\
\text { height }(\mu \mathrm{m}) / \mathrm{In} \\
\text { content }(\%)\end{array}$ \\
\hline $\mathrm{B} 1^{19}$ & $\mathrm{Si}(111)$ & $\cdots$ & $\sim 0.5 / \sim 25$ \\
\hline Ref. SA NCs & & & \\
\hline $\begin{array}{l}\mathrm{B} 2^{20} \\
\mathrm{SAG} N C s\end{array}$ & GaN-on-Si(111) & 0.5 & $\sim 0.5 / \sim 15$ \\
\hline $\mathrm{B}^{20}$ & GaN-on-Si(111) & 0.5 & $\sim 0.5 / \sim 25$ \\
\hline SAG NCs & & & \\
\hline $\begin{array}{l}\mathrm{B} 4^{20} \\
\mathrm{SAGNCs}\end{array}$ & GaN-on-Si(111) & 0.2 & $\sim 0.4 / 100$ \\
\hline
\end{tabular}

\section{B. Ordered ensembles of $\operatorname{In}(\mathrm{Ga}) \mathrm{N}$ nanowires}

Crystalline properties of a reference SA InGaN/GaN NC sample, grown on bare $\mathrm{Si}(111)$ (sample B1, details about this sample can be found in our previous publication $)^{19}$ is compared with SAG $\operatorname{In}(\mathrm{Ga}) \mathrm{N} / \mathrm{GaN}$ NC samples grown on
GaN-on-Si(111) templates (B2, B3, and B4, details about these samples can be found in our previous publication). ${ }^{20}$ The most relevant data about the analyzed samples, which are columnar InGaN: height and composition, are summarized in Table II.

\section{SA vS. SAG: Site-, size-, and orientation-control}

Figure 5 shows lateral and top SEM view of the InGaN/ GaN NCs, grown by: (B1) SA heteroepitaxy on bare $\mathrm{Si}(111)^{19}$ and (B2-B4) SAG on GaN-on-Si(111) templates. ${ }^{20}$ Observe that unlike SAG GaN NCs, which are fabricated fully homoepitaxially in one growth step, the fabrication of $\mathrm{SAG} \mathrm{In}(\mathrm{Ga}) \mathrm{N} / \mathrm{GaN} \mathrm{NCs}$ requires two growth steps and involves heteroepitaxy. Consequently, for the sake of precision, we refer to the latter fabrication method simply as SAG (instead of SAG homoepitaxy).

The first growth step concerns growth of the underlying columnar GaN section. This is achieved through SAG homoepitaxy on the underlying GaN-on-Si template, as explained in Sec. III A. ${ }^{5}$ The second growth step concerns growth of the top In $(\mathrm{Ga}) \mathrm{N}$ columnar section. The growth conditions

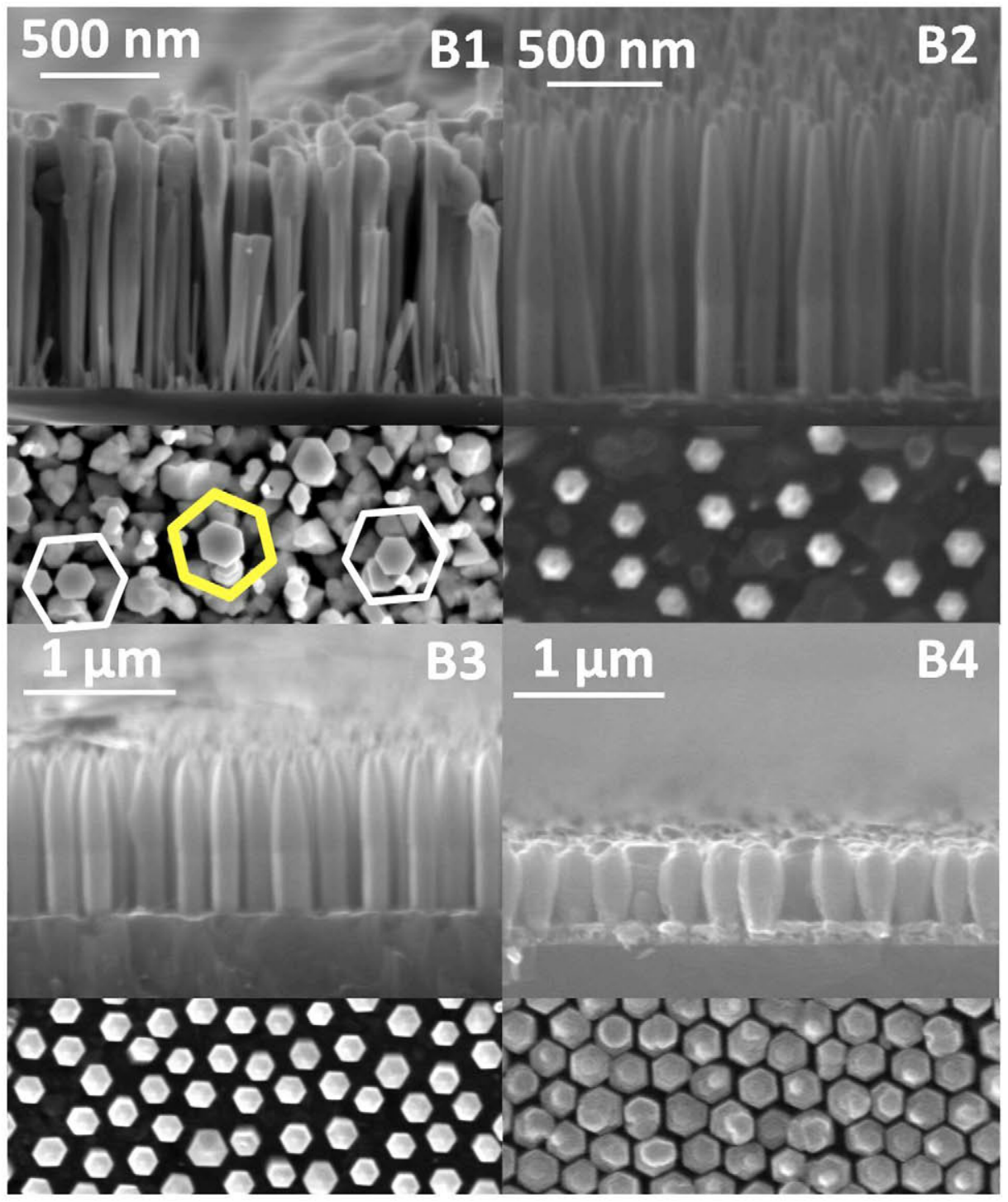

FIG. 5. SEM images of $\operatorname{In}(\mathrm{Ga}) \mathrm{N} / \mathrm{GaN}$ NC ensembles (samples (B1)(B4)). ${ }^{19,20}$ (B1) The top view reveals that SA NCs grow with two different twist orientations (types A and B). (B2)-(B4) The top view reveals that SAG NCs grow with single twist orientation (type A). 
that are typically used for this second growth step are explained in our previous publications. ${ }^{19,20}$

SEM images of samples B1-B4 show that InGaN NCs fabricated by SAG (B2-B4) exhibit: (i) order, (ii) size uniformity, and (iii) crystallographic uniformity, in respect to their SA counterpart (B1). Note that the NC positioning (i) and size uniformity (ii) are achieved through the same means of the SAG homoepitaxy in the first growth step, as previously described for the case of SAG GaN NCs. On the other hand, the appearance of the columnar $\operatorname{In}(\mathrm{Ga}) \mathrm{N} / \mathrm{GaN}$ heterointerface, which is not present in the case of GaN SAG NCs, can have significant impact on the ensemble crystallographic uniformity (iii). In this section, we provide a detailed insight into the ensemble crystallographic and compositional uniformity, through a comparative study of SA and SAG NCs.

\section{Crystallographic uniformity}

Figure 5(B1) reveals that SA NCs grow with two different twist orientations: (a) type A (the dominant one) and (b) type $\mathrm{B}$, rotated $30^{\circ}$ with respect to the dominant one. On the other hand, in all samples grown by SAG, the NCs have single twist orientation.

All samples under study (B1-B4) have two heterointerfaces:

(i) GaN-SiN-Si(111) heterointerface, which is characterized by a lack of a direct crystal-to-crystal contact (weak epitaxial constraint). In sample B1, this is a column-to-bulk heterointerface, whereas in samples B2-B4, this is a bulk heterointerface.

(ii) $\operatorname{In}(\mathrm{Ga}) \mathrm{N}-\mathrm{GaN}$ heterointerface which is characterized with a direct crystal-to-crystal contact. In all samples, this is a columnar heterointerface.

The coexistence of two different NC twists in sample B1, is a fingerprint of two different epitaxial relationships, at either GaN-SiN-Si(111) or InGaN-GaN heterointerface. Bearing in mind that the first GaN-SiN-Si(111) heterointerface is characterized by a weak epitaxial constraint, that the second columnar InGaN-GaN heterointerface is identical in all studied samples, and that all samples B2 to B4 exhibit single crystallographic twist, the origin of two different epitaxial relationships in sample B1 has been linked to the first heterointerface. This result is not surprising, since it is known that GaN NCs can nucleate on the underlying $\mathrm{Si}(111)$ with two different twists, that is: ${ }^{32}$

(a) $\mathrm{GaN}(10-10) \| \mathrm{Si}(112)$, type $\mathrm{A}$ (the dominant one)

(b) $\mathrm{GaN}(11-20) \| \mathrm{Si}(112)$, type B.

The frequency of type B alignment is influenced mainly by the $\mathrm{Si}(111)$ substrate miscut and growth conditions in the initial growth stage. In our case, we find the incidence of this alignment significantly below $1 \%$.

The question of crystallographic uniformity of SAG InGaN NCs is more complicated than that of their SAG GaN NC counterparts, described in Sec. III A. This is mainly because in this system we deal with one additional heterointerface (of columnar type), which is not present in the former case. The $\operatorname{In}(\mathrm{Ga}) \mathrm{N}$ NCs are epitaxially aligned to the underlying $\mathrm{Si}(111)$ substrate, however, in the present case the degree of their alignment is "double-disturbed," since each of the two heterointerfaces can produce certain crystal lattice tilting and/or twisting.

a. Epitaxial relationship analysis. To determine the epitaxial relationship observed in the considered SAG NC ensembles with certainty, we performed XRD studies. For these studies, we selected sample B4, since having its topcolumnar section composed of a binary $(\mathrm{InN})$ material, this sample yields more diffracting signal than its ternary counterparts (B2 and B3). For XRD measurements, the samples were aligned according to the $\mathrm{GaN}$ buffer crystal planes, making use of (0002) and (10-13) Bragg spots (to set the reference zero-tilt and zero-twist values, respectively). The signal was recorded with a fully open detector. The obtained results are shown in Figure 6.

Figure 6(a) shows a $\omega-2 \Theta / \Delta \omega$ map, revealing a "tiltrelated" epitaxial relationship of $\mathrm{InN}, \mathrm{GaN}$, and $\mathrm{Si}$ crystal planes. This figure reveals a following effect:

Effect I: The mean $\Delta \omega$-value of $\operatorname{InN}(0002)$ and $\mathrm{GaN}(0002)$ Bragg reflections is different than that of $\mathrm{Si}(111)$ Bragg reflections, indicating the growth of a slightly crystallographically rotated $\mathrm{InN} / \mathrm{GaN}$ epilayer (tilt-related) on the underlying Si substrate.

This slight crystallographic rotation of the epilayer with respect to the $\mathrm{Si}$ substrate is estimated at $\Varangle(G a N(0001)$, $\operatorname{Si}(111)) \sim 2.2^{\circ}$. When epitaxial growth is performed on $\mathrm{Si}(111)$ substrates with $\sim 4^{\circ}$ miscut, we find the grown III-N epilayer typically 1 to $3^{\circ}$ rotated with respect the underlying substrate. These values are higher than the value predicted by Nagai et al. $\left(\sim 0.7^{\circ}\right),{ }^{33}$ and in agreement with recent experimental values, reported by Wang et al. ${ }^{34}$ On the other hand, no crystallographic tilt is observed at the InN/GaN columnar heterointerface, meaning that the columnar InN planes closely follow the alignment of the underlying columnar $\mathrm{GaN}$ : $\operatorname{InN}(0001) \| \mathrm{GaN}(0001)$.

Figure 6(b) shows $\omega-2 \Theta / \varphi$ pole map, revealing a "twistrelated" epitaxial alignment of $\mathrm{InN}, \mathrm{GaN}$ and $\mathrm{Si}$ crystal planes. The pole map confirms a three-fold symmetry of the $\mathrm{Si}(220)$ Bragg reflection. It further reveals a six-fold symmetry of the $\mathrm{GaN}$ and $\mathrm{InN}$ layers grown on top, which originates from the wurtzite structure of the III-nitride unit cells. The measurement reveals a $\mathrm{Si}(11-2)|| \mathrm{GaN}(10-10)|| \operatorname{InN}(10-10)$ "twist-related" epitaxial relationship, the one corresponding to type A orientation.

For the sake of precision, let us note that this pole map is a sum of two $\omega-2 \Theta / \varphi$ pole figures, recorded around (i) $\mathrm{Si}(220)$ Bragg reflection (with $\psi=35.26^{\circ}$ ) and (ii) GaN(10-13) Bragg reflection (with $\psi=32.04^{\circ}$ ), respectively. The two pole figures were recorded over the same $\omega /$ $2 \Theta$ and $\varphi$ ranges $\left(\omega / 2 \Theta=18^{\circ}-38^{\circ} / 36^{\circ}-72^{\circ}\right.$ and $\varphi=0-360^{\circ}$, respectively), as well as with the same reference $\omega_{0} / \varphi_{0}$ angles. Note that due to similar $\psi$ angle values for $\mathrm{GaN}(10-13)$ and $\operatorname{InN}(10-13)\left(32.04^{\circ}\right.$ and $31.86^{\circ}$, respectively) these two Bragg reflections were recorded together (in the second scan). This approach permits the sum of two pole figures to be presented as a single pole map. 

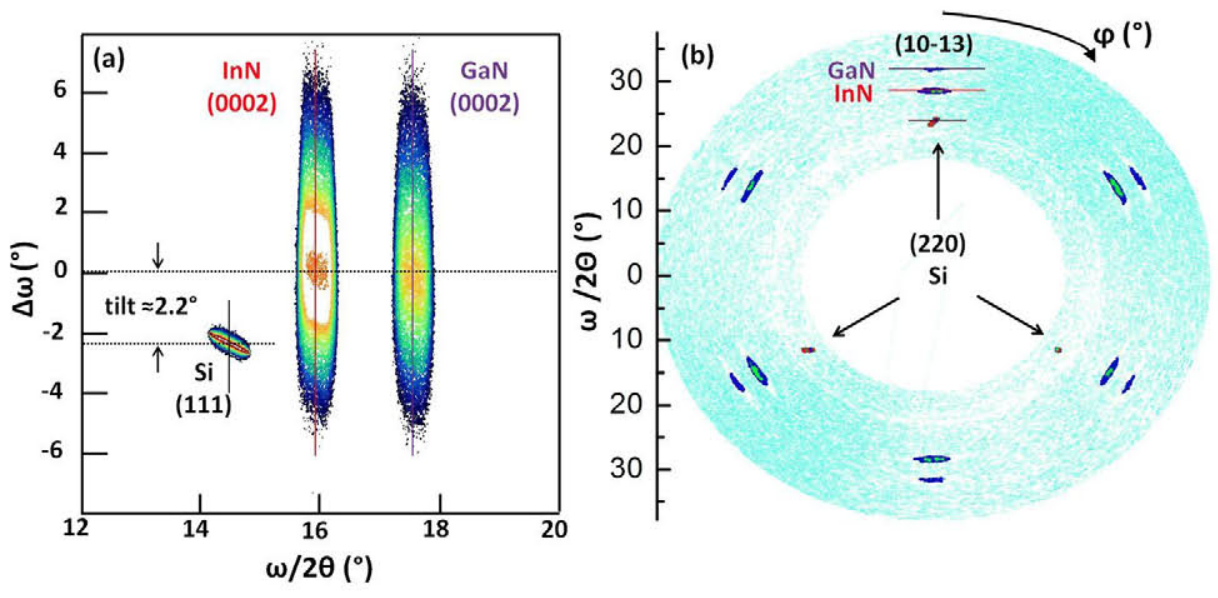

FIG. 6. (a) $\omega-2 \Theta / \Delta \omega$ map, for sample B4, reveals a $\operatorname{InN}(0001) \| \mathrm{GaN}(0001)$ ||Si(111) "tilt-related" epitaxial relationship, modified with a slight crystallographic tilt $\left(\sim 2.2^{\circ}\right)$ at the epilayersubstrate heterointerface. (b) $\omega-2 \Theta / \varphi$ pole map reveals $\mathrm{InN}(10-10)|| \mathrm{GaN}(10-$ 10) ||Si(112) "twist-related" epitaxial relationship. The observed epitaxial relationship corresponds to type A orientation. b. Tilt and twist analysis. Apart from effect I observed in Figure 6(a), both Figs. 6(a) and 6(b) reveal one more important effect:

Effect II-a (tilt-related $(\Delta \omega)$ ): The width of $\operatorname{InN}(0002)$ and $\mathrm{GaN}(0002)$ Bragg reflections is significantly higher than that of Si(111) Bragg reflection (Fig. 6(a)).

Effect II-b (twist-related $(\varphi)$ ): The width of $\operatorname{InN}(10-13)$ and $\mathrm{GaN}(10-13)$ Bragg reflections is significantly higher than that of Si(220) Bragg reflection (Fig. 6(b)).

These results indicate that the crystallographic uniformity of the InN-GaN epilayer is inferior in respect to crystallographic uniformity of $\mathrm{Si}$ substrate, as expected.

To get quantitative insight into a degree of crystallographic uniformity, we assessed an average tilt and twist of each of the three crystal layers ( $\mathrm{InN}, \mathrm{GaN}$, and $\mathrm{Si}$ ) in sample B4. The average tilts are defined as one half of full width at half maximum $(1 / 2 \cdot \mathrm{FWHM})$ of $\omega$-rocking scans performed around the $\mathrm{InN}(0002)$, $\mathrm{GaN}(0002)$, and $\mathrm{Si}(111)$ Bragg reflections, whereas the average twists are defined as $1 / 2$.FWHM of $\varphi$-scans performed around the $\mathrm{InN}(10-13), \mathrm{GaN}(10-13)$ and $\mathrm{Si}(220)$ Bragg reflections, respectively. Note that these $1 \mathrm{D}$ scans correspond to $\Delta \omega$ - and $\varphi$-extracts along the solid lines designated in Figs. 6(a) and 6(b), respectively. The $\omega$ rocking and $\varphi$-scans are featured in Figures 7(a) and 7(b), whereas the average tilts and twist, extracted from them, are summarized in Figure 7(c).

The measurements reveal a drastic increase of average tilt and twist values at the bulk GaN-SiN-Si heterointerface (around 500 times). No further increase is observed at the columnar InN-GaN heterointerface. Note that the latter result is in a qualitative agreement with SEM images shown in Figure 5, since the orientation of the top $\mathrm{NC}$ part in respect to the bottom one, does not seem to be disturbed by the presence of the intra-columnar heterointerface (Figure 5). When interpreting average tilt/twist values (estimated by XRD), one should bear in mind that unlike RHEED which excites the crystal surface only, the XRD technique excites the entire crystal volume. Consequently, the retrieved $\mathrm{Si}, \mathrm{GaN}$ and $\mathrm{InN}$ tilt/twist values are averaged over entire $\mathrm{Si}, \mathrm{GaN}$ and $\mathrm{InN}$ material, contained in the analyzed sample. In the case of $\mathrm{GaN}$, it retrieves information about bulk + columnar $\mathrm{GaN}$. Since the measurements were performed with a fully open detector to increase the number of counts, we cannot exclude a possible contribution of parasitic $\mathrm{GaN} / \mathrm{InN}$ material to the recorded XRD spectra. This parasitic material may nucleate on the underlying TiN during the SAG.

In the end of this subsection, it is worth to note that both: (i) epitaxial relationship analysis and (ii) tilt/twist analysis revealed significant differences between the bulk GaN$\mathrm{SiN}-\mathrm{Si}$ and columnar In(Ga)N-GaN heterointerfaces. The former heterointerface is, on one hand, characterized by a slight tilt-related crystallographic rotation of the epilayer with respect to the substrate (found to be $\sim 2^{\circ}$ in the case of sample B4, effect I) and, on the other hand, by a drastic decrease in epilayer crystallographic uniformity with respect to that of the underlying substrate (found to be $\sim 500$ in the analyzed case, effect II). Neither of the two features is observed at the second heterointerface (crystallographic rotation is $\sim 0^{\circ}$ and a decrease in crystallographic uniformity is $\sim 1)$. These differences are mainly considered fingerprints of an indirect crystal contact ( $\mathrm{GaN}-\mathrm{SiN}-\mathrm{Si})$ at the first heterointerface (a weak epitaxial constraint) and a direct crystal contact (InN-GaN) at the second heterointerface, respectively.

\section{Compositional uniformity}

In the recent years, several groups reported that spectral purity of InGaN NC ensemble luminescence, improves drastically when the growth method changes from SA to SAG. ${ }^{6-8,14-16}$ These results strongly suggest that the SAG yields ensembles with improved compositional uniformity. It is worth to notice that the optical techniques (such as PL, cathodoluminescence, and/or electroluminescence) assess the recombination of photo-carriers generated in the crystal upon excitation. Consequently, they mainly yield information about high crystal quality regions with somewhat higher In content, due to suppressed non-radiative and enhanced radiative recombination, in these regions. In addition, the heterointerfaces of III-Ns are characterized by strong electric fields, which can significantly alter the electron-hole recombination energy and lead to heavy misinterpretations, when compositional analyses are concerned.

To avoid possible misinterpretations, in this work we employ a structural XRD technique. Unlike optical techniques, which retrieve compositional information making use of photo-carriers generated in the crystal upon excitation, the XRD assesses the crystal lattice directly, by means of 

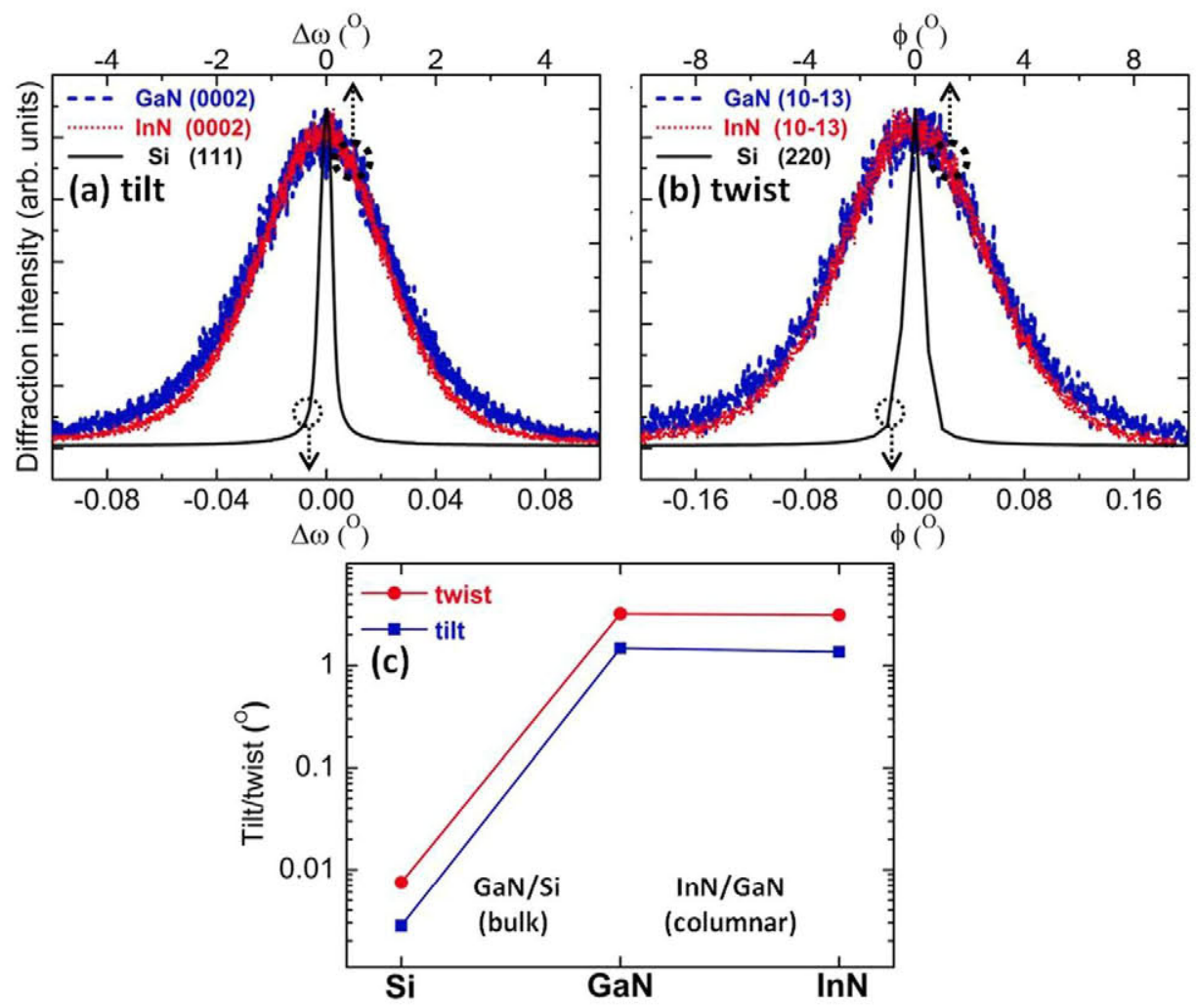

FIG. 7. (a) w-rocking curves and (b) $\varphi$-scans show that (c) the corresponding FWHM values increase $\sim 2.5$ orders of magnitude at the $\mathrm{GaN} / \mathrm{Si}$ heterointerface. No further increase is observed at the InN/GaN columnar heterointerface. For $\mathrm{GaN}$ and $\mathrm{InN} \omega$ rocking and $\varphi$-scan curves, the top axis is used, whereas for Si curves the bottom axis (dilated 50 times) is used, for clarity. diffraction. Apart from the ternary composition, the properties of the InGaN NC crystal lattice are strongly influenced by strain. ${ }^{13}$ To eliminate potential sources of systematic errors, we also assess average strain of NCs under study. The XRD measurements are complemented with macro-PL measurements, for completeness. Both techniques yield information averaged over $\sim 1 \mathrm{~mm}^{2}$ sample area.

a. XRD analysis. The average In content and compositional uniformity of columnar InGaN sections were studied by $\omega / 2 \Theta$ scans around the (0002) GaN Bragg reflection (Fig. 8(a), samples B1-B4). The signal was recorded with the $\mathrm{Ge}(220)$ hybrid monochromator placed in front of the detector.

A wide, Gaussian-like intensity distribution, recorded in the case of the reference SA NC sample (B1) is an indication of a poor compositional uniformity, which originates from a high randomness of the SA growth method. ${ }^{13}$ Relatively narrow, Lorentzian-like InGaN diffraction peaks are found over entire InGaN compositional range on SAG NC ensembles (samples B2-B4). A narrow, Lorentzian-like distribution is an indication of a good compositional uniformity. ${ }^{13}$ Their FWHM values are comparable to those of single InGaN 2D layers, grown on a commercial $c$-plane $\mathrm{GaN}$-on-sapphire pseudosubstrate, under optimal intermediate metal-rich conditions with a metallic In bilayer on the growth front. ${ }^{21}$ Figure $8(\mathrm{a})$ includes $\omega / 2 \Theta$ scan of $2 \mathrm{D}$ InGaN layer, $\sim 100 \mathrm{~nm}$ thick and with the same targeted In content $(\sim 25 \%)$ like in B1 and B3 samples, for comparison (growth details about this sample can be found in our previous publication). ${ }^{21}$

The position of InN peak (sample B4 in Figure 8(a)) reveals that the nanocolumnar InN grows nearly fully relaxed. To assess the strain in nanocolumnar InGaN sections, reciprocal space mapping was taken around the (10-15) GaN Bragg reflection. The mapping reveals that with increasing In content $(\sim 15, \sim 25$, and $100 \%)$ the InGaN (10-15) diffraction peak displaces along the trajectory that corresponds to that of the fully relaxed InGaN. Figure 8(b), shows the measurement for sample B3. The average strain is found to be below $1 \%$, for all three samples.

b. PL analysis. According to XRD experimental evidence, the compositional uniformity of the InGaN columnar sections improves significantly when they are grown by SAG. This improvement has strong consequences on the PL spectral shape, allowing for higher uniformity of the NC ensemble emission. Figure 9 shows a comparison of PL spectra (normalized) of SA and SAG NC samples (B1 and B3), both having the same targeted In content of $\sim 25 \%$. $^{19,20}$ The corresponding FWHM values, estimated to be 0.54 and $0.15 \mathrm{eV}$, respectively, point to significantly improved luminescence spectral purity, in the SAG case. ${ }^{19,20}$ The B3 sample emission, is characterized by a clear and bright green emission (for details, see Ref. 20). This result is in a qualitative agreement with electroluminescence properties of ensembles of SA InGaN/GaN NCs, reported by Bavencove et al., ${ }^{16}$ and those of SAG InGaN/GaN NCs reported by BengoecheaEncabo et $\mathrm{al}^{8}{ }^{8}$ In the former case, the authors reported pronounced spotty and polychromatic emission. No similar effects have been observed in the latter case. In addition, a $40 \%$ improvement of the FWHM of the ensemble electroluminescence (from $\sim 360$ down to $\sim 220 \mathrm{meV}$ ) was reported for green nano-LEDs, when the growth method changed from SA to the SAG one. ${ }^{8,16}$ The narrowing of the $\mathrm{NC}$ ensemble PL by the means of SAG, facilitates fabrication of 


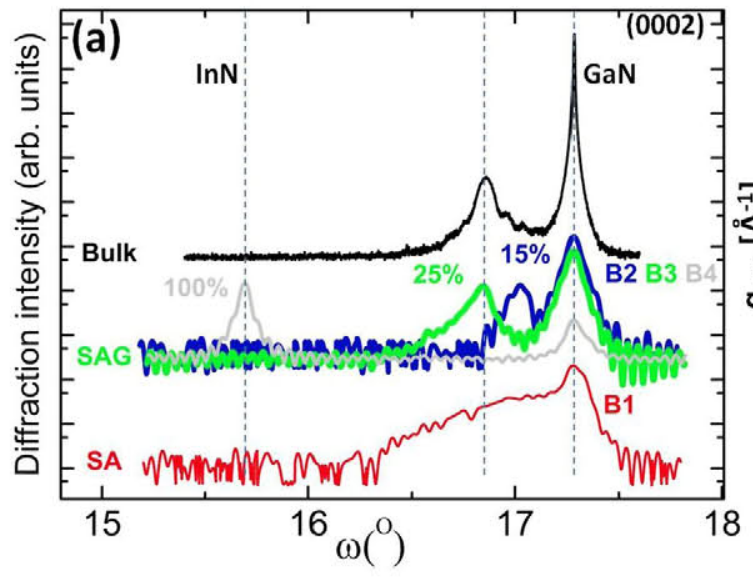

arrays of single color emitting nano-LEDs over entire visible range. Details about the realization of blue, green, and yellow nano-LEDs can be found in our separate publication. ${ }^{8}$

\section{DISCUSSION}

Due to its high importance for practical applications, several approaches to improve NC ensemble crystallographic uniformity have been studied. In this discussion, we will focus exclusively on those reported for GaN NCs grown on $\operatorname{Si}(111){ }^{16,32,35-37}$

Three different types of GaN NCs to Si(111) alignment have been reported in the literature:

(a) single alignment, with type A epitaxial relationship $(\mathrm{GaN}(10-10)|| \operatorname{Si}(11-2)),{ }^{35}$

(b) coexistence of two different alignments, type $\mathrm{A}$ and type $\mathrm{B}(\mathrm{GaN}(11-20)|| \mathrm{Si}(11-2))$ epitaxial relationships ${ }^{32}$

(c) random alignment (no epitaxial relationship). ${ }^{35,36}$

The strategies for improvement can be roughly divided into two groups:

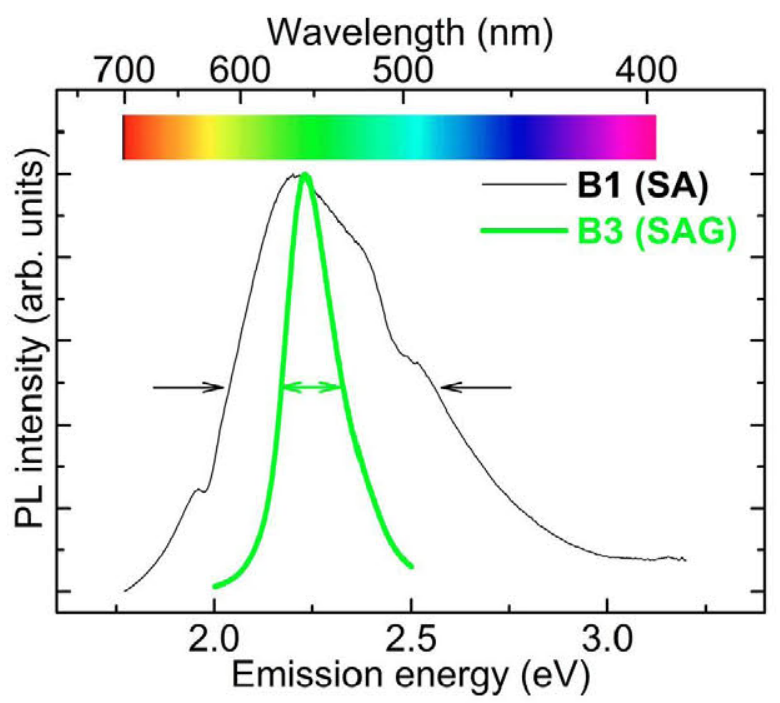

FIG. 9. Comparison of PL spectra at $10 \mathrm{~K}$ (normalized) of ordered $\mathrm{InGaN} /$ GaN NCs (sample B3) with that of SA InGaN/GaN NCs (sample B1), both emitting in the green. The FWHM improvement (from 0.54 to $0.15 \mathrm{eV}$ ) for the SAG NCs is attributed to its better morphological uniformity. ${ }^{19,20}$ (i) optimization of growth conditions

(ii) optimization of the interlayer lying between $\mathrm{GaN}$ NCs and $\mathrm{Si}(111)$ substrate.

Regarding (i), Wierzbicka et al. ${ }^{35}$ recently reported that the GaN NCs to $\mathrm{Si}(111)$ alignment is particularly sensitive to the initial nitridation of the $\mathrm{Si}(111)$ surface. Making use of XRD pole figures, the authors demonstrated that decreasing temperature of the initial nitridation step led to a progressive deterioration of the NC-to-substrate alignment; when the initial nitridation was performed at common growth temperature $\left(800^{\circ} \mathrm{C}\right.$ ), single alignment (type A) was observed, whereas when it was performed at low temperatures $\left(150^{\circ} \mathrm{C}\right)$, the epitaxial relationship was fully lost, resulting with a random alignment. ${ }^{35}$ These results suggest that depending on the properties of the amorphous $\mathrm{SiN}$ interlayer, it can allow or fully block the transfer of epitaxial information, from the underlying $\mathrm{Si}(111)$ substrate to the GaN NCs epilayer.

Regarding (ii), several approaches related to the substitution of the amorphous $\mathrm{SiN}$ interlayer have been reported. First, let us note that its substitution with other amorphous materials, such as amorphous $\mathrm{Al}_{2} \mathrm{O}_{3}$, gave similar or worse results. Sobanska et al. reported that no matter how thick $\mathrm{Al}_{2} \mathrm{O}_{3}$ amorphous interlayer is, it always blocks the transfer of the epitaxial information through $\mathrm{GaN}(0001)-\mathrm{Al}_{2} \mathrm{O}_{3}$ $\mathrm{Si}(111)$ heterointerface. ${ }^{36}$ Better results were reported when the amorphous SiN interlayer was substituted with crystalline interlayers such as AlN or AlGaN. ${ }^{16,32,37}$ The presence of $\mathrm{Al}$ on the $\mathrm{Si}(111)$ surface prevents the formation of amorphous SiN, leading to the formation of entirely crystalline $\mathrm{GaN}(0001)-\mathrm{Al}(\mathrm{Ga}) \mathrm{N}-\mathrm{Si}(111)$ heterointerface. Although several authors reported qualitative improvement in the NC-tosubstrate alignment in this case (attributed mainly to the direct crystalline contact), ${ }^{16,37}$ close analysis by the combination of SEM images and XRD pole figures revealed coexistence of type A and type B alignments. ${ }^{32}$ Similarly to the findings of Wierzbicka et al.,$^{35}$ the authors pointed out that the growth conditions had significant influence on the NCs alignment and that by optimizing them, the weight of the dominant alignment could be increased to $\sim 98 \% .^{32}$ In summary, the previous studies report that the GaN NCs-to$\mathrm{Si}(111)$ alignment is sensitive to both growth conditions and employed interlayer ( $\mathrm{SiN}, \mathrm{Al}_{2} \mathrm{O}_{3}, \mathrm{AlN}$, and $\mathrm{AlGaN}$ ) and 
varies from a weak single epitaxial relationship (at best) to no epitaxial relationship (at worst).

Our results are in agreement with the previous findings. When growing SA GaN NCs on an unintentionally nitridated $\mathrm{Si}(111)$ ( $\mathrm{Si}$ is spontaneously nitridated in the early growth stage, at $\sim 800^{\circ} \mathrm{C}$ ) we typically observe type A alignment, with a very sporadic appearance of type B alignment. By careful SEM inspections, B alignment was found to have a weight of less than $1 \%$ (Fig. 5(a)).

The recently reported formation mechanisms for SA GaN NCs, suggest that their self-nucleation may be intrinsically incompatible with their high crystallographic uniformity. ${ }^{22}$ Namely, it is the combination of the huge lattice mismatch to the underlying substrate and highly N-rich conditions that has been identified as the driving mechanism leading to their self-nucleation. When combined with the small NC-to-substrate interface (typically $<100 \times 100 \mathrm{~nm}^{2}$ ), these conditions seem to lead to a significant random tilting and twisting of the initial nucleation seeds, and consequently, to a poor crystallographic uniformity of the $\mathrm{NC}$ ensemble developed from them in the posterior growth stage.

The problem of poor initial crystallographic uniformity can be circumvented by homoepitaxy on $\mathrm{GaN}$ templates. Due to the lack of the GaN/substrate lattice mismatch in this case, the homoepitaxy is intrinsically incompatible with the self-nucleation mechanism, as reported by Consonni et al. To surmount this obstacle, the SAG method, assisted by a lithographic mask in its very initial growth stage, has been developed. The formation mechanism for SAG GaN NCs in this case, is substantially different from that reported for SA GaN NCs, and can be found in our separate publication. ${ }^{23}$

In the case of GaN NCs, fabricated by this method, both SEM inspections and XRD pole figures reveal single type A alignment (Figs. 1, 5, and 6). Notice that the growth of GaN buffers on either Si or sapphire substrates leads to the formation of a single GaN crystal. When GaN NC nucleation is started homoepitaxially on a single crystal seed, the final NCto-substrate alignments are unambiguously determined by the crystal orientation of the underlying substrates. For $\mathrm{Si}(111)$ and sapphire(0001), we find "twist-related" epitaxial relationship to be $\mathrm{GaN}(10-10) \| \mathrm{Si}(11-2)$ and $\mathrm{GaN}(10-10)||$ sapphire (11-20). Concerning "tilt-related" epitaxial relationship, it is found as commonly reported: $\mathrm{GaN}(0001) \| \mathrm{Si}(111)$ and $\mathrm{GaN}(0001)||$ sapphire(0001), corrected with a slight tilt-related crystallographic rotation, which can emerge at the GaN/Si heterointerface. The angle of this slight crystallographic rotation is smaller than the angle of the substrate miscut, i.e., it is typically of the order of $\sim 1^{\circ}$ or smaller, and can be considered irrelevant for practical applications.

To improve the crystallographic uniformity of the GaN NCs grown on $\mathrm{Si}$, note that we actually do not proceed to the removal of the amorphous $\mathrm{SiN}$ interlayer, which is still present at the GaN-Si heterointerface. Instead, by employing nearly stoichiometric growth conditions for the GaN buffer growth, we first reduce the number of those crystal seeds that nucleate with any other but the dominant type A alignment. Second, by forcing their coalescence into a compact GaN layer, we hinder growth of those crystal seeds that nucleated with any other but the dominant type A alignment (at expense of dislocations formation in the compact layer itself). In this way, a single crystal GaN buffer layer is formed. Instead of growing the NCs on the heterointerface itself (SA approach), the amorphous $\mathrm{SiN}$ interlayer is buried below the compact single crystal GaN buffer, and the NC nucleation is started directly on it by the means of SAG homoepitaxy.

\section{CONCLUSION}

In summary, in this article we gave a systematic insight into crystallographic and compositional uniformity of the SAG (In)GaN NC ensembles. First, making use of SEM, RHEED, and XRD techniques, we showed that SAG homoepitaxy, apart from NC site- and size-control, also leads to improved NC orientation-control. As a consequence, the SAG NCs exhibit single epitaxial relationship to the underlying $\mathrm{Si}(111)$ and sapphire(0001) substrates. Second, making use of the combination of XRD and macro-PL techniques, we showed that the combination of site-, size-, and orientationcontrol leads to improved compositional uniformity of $\mathrm{InGaN}$ $\mathrm{NC}$ ensembles. These two improvements facilitate $\mathrm{NCs}^{\prime}$ integration into devices as well as their employment in singlecolor applications, opening the door for the realization of next-generation NC-based III-N optoelectronic devices.

\section{ACKNOWLEDGMENTS}

We acknowledge N. García-Lepetit and P. E. D. Soto Rodriguez from ISOM-UPM, Madrid, for help in epitaxial growth and data processing, and Professor M. A. SanchezGarcia for discussions and help in numerous technical issues. This work was partially supported by research grants: EU FP7 Contract No. GECCO 280694-2 and the Spanish Project Nos. CAM/P2009/ESP-1503 and MICINN MAT201126703.

${ }^{1}$ M. Yoshizawa, A. Kikuchi, M. Mori, N. Fujita, and K. Kishino, Jpn. J. Appl. Phys., Part 2 36, L459 (1997).

${ }^{2}$ M. A. Sánchez-García, E. Calleja, E. Monroy, F. J. Sánchez, F. Calle, E. Muñoz, and R. J. Beresford, J. Cryst. Growth 183, 23 (1998).

${ }^{3}$ H. Sekiguchi, K. Kishino, and A. Kikuchi, Appl. Phys. Express 1, 124002 (2008).

${ }^{4}$ K. Kishino, H. Sekiguchi, and A. Kikuchi, J. Cryst. Growth 311, 2063 (2009).

${ }^{5}$ A. Bengoechea-Encabo, F. Barbagini, S. Fernández-Garrido, J. Grandal, J. Ristić, M. A. Sánchez-GarcSáncheza, E. Calleja, U. Jahn, E. Luna, and A. Trampert, J. Cryst. Growth 325, 89 (2011).

${ }^{6} \mathrm{~K}$. Kishino, J. Kamimura, and K. Kamiyama, Appl. Phys. Express 5, 031001 (2012).

${ }^{7}$ K. Kishino, K. Nagashima, and K. Yamano, Appl. Phys. Express 6, 012101 (2013).

${ }^{8}$ A. Bengoechea-Encabo, S. Albert, D. Lopez-Romero, P. Lefebvre, F. Barbagini, A. Torres-Pardo, J. M. Gonzalez-Calbet, M. A. SanchezGarcia, and E. Calleja, Nanotechnology 25, 435203 (2014).

${ }^{9}$ E. Chernisheva, S. Lazić, Ž. Gačević, N. García-Lepetit, H. P. van Muelen, M. Müller, F. Bertram, P. Veit, A. Torres-Pardo, J. M. González Calbet, J. Christen, E. Calleja, and J. Manuel Calleja, "Blue-to-green nonclassical light emission from site-controlled GaN nanowires hosting InGaN nanodisks," Opt. Express (submitted).

${ }^{10}$ K. J. Kuhn, Microelectron. Eng. 88, 1044 (2011).

${ }^{11} \mathrm{~S}$. Albert, A. Bengoechea-Encabo, J. Zuniga-Perez, P. de Mierry, P. Val, M. A. Sanchez-Garcia, and E. Calleja, Appl. Phys. Lett. 105, 091902 (2014).

${ }^{12}$ H. Matsubara, S. Yoshimoto, H. Saito, Y. Jianglin, Y. Tanak, and S. Noda, Science 319, 445 (2008). 
${ }^{13}$ M. A. Moram and M. E. Vickers, Rep. Prog. Phys. 72, 036502 (2009).

${ }^{14}$ K. Kishino, A. Kikuchi, H. Sekiguchi, and S. Ishizawa, Proc. SPIE 6473, 64730T (2007).

${ }^{15}$ H.-W. Lin, Y.-J. Lu, H.-Y. Chen, H.-M. Lee, and S. Gwo, Appl. Phys. Lett. 97, 073101 (2010).

${ }^{16}$ A. L. Bavencove, G. Tourbot, J. Garcia, Y. Désières, P. Gilet, F. Levy, B. André, B. Gayral, B. Daudin, and L. S. Dang, Nanotechnology 22, 345705 (2011).

${ }^{17}$ Y. Nakada, I. Aksenov, and H. Okumura, Appl. Phys. Lett. 73, 827 (1998).

${ }^{18}$ A. Bengoechea-Encabo, S. Albert, M. A. Sanchez-Garcia, L. L. López, S. Estradé, J. M. Rebled, F. Peiró, G. Nataf, P. de Mierry, J. Zuniga-Perez, and E. Calleja, J. Cryst. Growth 353, 1 (2012).

${ }^{19}$ S. Albert, A. Bengoechea-Encabo, P. Lefebvre, M. A. Sánchez-Garcca, E. Calleja, U. Jahn, and A. Trampert, Appl. Phys. Lett. 99, 131108 (2011).

${ }^{20} \mathrm{~S}$. Albert, A. Bengoechea-Encabo, M. A. Sánchez-García, X. Kong, A. Trampert, and E. Calleja, Nanotechnology 24, 175303 (2013).

21 Ž. Gačević, V. J. Gómez, N. García-Lepetit, P. E. D. Soto Rodríguez, A. Bengoechea-Encabo, S. Fernández-Garrido, R. Nötzel, and E. Calleja, J. Cryst. Growth 364, 123 (2013).

${ }^{22}$ V. Consonni, M. Hanke, M. Knelangen, L. Geelhaar, A. Trampert, and H. Riechert, Phys. Rev. B 83, 035310 (2011).

23̌̌. Gačević, D. Gómez Sánchez, and E. Calleja, "Formation Mechanisms of GaN Nanowires Grown by Selective Area Growth Homoepitaxy," Nano Lett. (submitted).

${ }^{24}$ R. Songmuang, O. Landré, and B. Daudin, Appl. Phys. Lett. 91, 251902 (2007).
${ }^{25}$ I. Horcas, R. Fernández, J. M. Gómez-Rodríguez, J. Colchero, J. GómezHerrero, and A. M. Baro, Rev. Sci. Instrum. 78, 013705 (2007).

${ }^{26} \mathrm{~S}$. Fernández-Garrido, V. M. Kaganer, C. Hauswald, B. Jenichen, M. Ramsteiner, V. Consonni1, L. Geelhaar, and O. Brandt, Nanotechnology 25, 455702 (2014).

${ }^{27} \mathrm{See}$ http://tools.lifetechnologies.com/content/sfs/COAPDFs/2014/1514878 S37492.pdf for the size dispersion of the polystyrene nanobeads used for the fabrication of colloidal nanohole masks.

${ }^{28}$ T. D. Moustakas, T. Lei, and R. J. Molnar, Physica B 185, 36 (1993).

${ }^{29}$ M. A. Reshchikov and H. Morkoç, J. Appl. Phys. 97, 061301 (2005).

${ }^{30}$ O. Brandt, C. Pfüller, C. Chèze, L. Geelhaar, and H. Riechert, Phys. Rev. B 81, 045302 (2010)

${ }^{31}$ P. Dogan, O. Brandt, C. Pfüller, A.-K. Bluhm, L. Geelhaar, and H. Riechert, J. Cryst. Growth 323, 418 (2011).

${ }^{32}$ L. Largeau, D. L. Dheeraj, M. Tchernycheva, G. E. Cirlin, and J. C. Harmand, Nanotechnology 19, 155704 (2008).

${ }^{33}$ H. Nagai, J. Appl. Phys. 45, 3789 (1974).

${ }^{34}$ L. Wang, F. Huang, Z. Cui, Q. Wu, Q. Liu, C. Zheng, Q. Mao, C. Xiong, and F. Jiang, Mater. Lett. 115, 89 (2014).

${ }^{35}$ A. Wierzbicka, Z. R. Zytkiewicz, S. Kret, J. Borysiuk, P. Dluzewski, M. Sobanska, K. Klosek, A. Reszka, G. Tchutchulashvili, A. Cabaj, and E. Lusakowska, Nanotechnology 24, 035703 (2013).

${ }^{36}$ M. Sobanska, A. Wierzbicka, K. Klosek, J. Borysiuk, G. Tchutchulashvili, S. Gieraltowska, and Z. R. Zytkiewicz, J. Cryst. Growth 401, 657 (2014).

${ }^{37}$ O. Landré, C. Bougerol, H. Renevier, and B. Daudin, Nanotechnology 20, 415602 (2009). 